\title{
Cooperative Binding by Cyclodextrin Dimers
}

\author{
Akira HARADA, Masaoki Furue, and Shun-ichi NoZAKURA \\ Department of Polymer Science, Faculty of Science, \\ Osaka University, Toyonaka, Osaka 560, Japan.
}

(Received August 15, 1979)

\begin{abstract}
Dimers containing $\beta$-cyclodextrin $(\beta$-CD) moieties at both ends of dicarboxylic acids were prepared by the reaction of bis $(m$-nitrophenyl) dicarboxylate with a large excess of $\beta$-CD, and were purified by dialysis and gel chromatography. The interaction of the dimers with potassium 2-p-toluidinylnaphthalene-6-sulfonate (TNS) was studied by the fluorescence technique and compared with that of $\beta-C D$ and poly(acryloyl- $\beta-C D)$. The dimers were found to bring about a much greater enhancement and blue shift of the TNS fluorescence than $\beta$-CD but less than the polymer. The succinate dimer enhanced the TNS fluorescence twofold more than the glutarate dimer. Dissociation constants and stoichiometries were determined by fluorometric titration and continuous variation methods. The dimer complexes showed exclusively $2: 1$ stoichiometry (CD:TNS), even in the concentration range in which $\beta$-CD showed $1: 1$ stoichiometry. The $2: 1$ complexes of the dimers were much more stable than the $2: 1$ complex of $\beta$-CD and were comparable to that of the polymer. Thus, two CD units linked by the chain were found to cooperate in binding one TNS molecule.

KEY WORDS Cyclodextrin / Cyclodextrin Dimer / Bis(cyclodextrin)

Succinate / Bis(cyclodextrin) Glutarate / 2-p-Toluidinylnaphthalene-6-sulfonate / Fluorescence / Binding / Inclusion /
\end{abstract}

Cyclodextrins (CD) are well known to form inclusion complexes with various molecules in aqueous solutions. The inclusion properties of $\mathrm{CDs}$ have been well investigated by a variety of physicochemical techniques and the results have been reviewed. ${ }^{1,2}$

Previously, we prepared polymers containing cyclodextrins in order to enhance the stability of complexes and to achieve better selectivity. ${ }^{3}$ Polymers containing $\beta$-CD were found to bind with large substrates which were too large to be accomodated in a single cavity more efficiently than $\beta$-CD. ${ }^{4}$ The polymer complexes showed exclusively $2: 1$ stoichiometry even in the concentration range where the complex of $\beta$-CD showed $1: 1$ stoichiometry. ${ }^{5}$ It is thought that the two adjacent $\beta$-CD moieties on a polymer chain cooperated in binding large substrates to the chain. These observation prompted us to examine the properties of $\mathrm{CD}$ dimers.

In this paper, we report on the preparation of bis$(\beta$-cyclodextrin) succinate and glutarate and their complexation properties. These dimers are abbreviated as $(\beta-C D)_{2} \mathrm{~S}$ and $(\beta-\mathrm{CD})_{2} \mathrm{G}$, respectively. The interaction of low molecular weight molecules with the dimers was studied by the fluorescence measure- ments, using potassium 2-p-toluidinylnaphthalene6-sulfonate (TNS) as a fluorescent probe. TNS is barely fluorescent in aqueous solutions but fluoresces strongly when adsorbed into certain hydrophobic binding sites. ${ }^{6,7}$ Data were compared with the case of $\mathrm{CD}$ and poly(acryloyl- $\beta-C D)^{5}$ to see whether or not the $C D$ moieties on the dimer behave independently or cooperatively in the binding of TNS and moreover to the details of this cooperation.

\section{RESULTS AND DISCUSSION}

\section{Preparation of Dimers}

$\operatorname{Bis}(\beta-C D)$ dicarboxylates were newly synthesized by the reaction of bis ( $m$-nitrophenyl) dicarboxylates with a large excess of $\beta-\mathrm{CD}$ at room temperature. In this procedure, one of the secondary hydroxyl groups of $\beta$-CD was transesterificated selectively under mild conditions, since this reaction proceeds by way of inclusion. ${ }^{8}$

The product was purified by dialysis or ultrafiltration using an Amicon UM-10 UF membrane to remove low molecular weight compounds (sodium chloride and $m$-nitrophenol) and by gel chromatog- 
raphy. A sephadex G-15 column was used to remove unreacted $\beta$-CD and biogel P-2 was used to remove monofunctional by-products. Yields were poor, probably because of the low solubility of $\operatorname{bis}(m-$ nitrophenyl) dicarboxylates in water. Both dimers obtained were chromatographically pure, and were characterized by NMR spectra, infrared spectra and elemental analysis as summarized in Table I. Molecular weights were confirmed by the vapor pressure osmometry.

\section{Fluorescence Change of TNS}

The fluorescence spectra of TNS in the presence of the dimers are shown in Figure 1 together with those in the presence of $\beta$-CD and the polymer. In this experiment the $\beta$-CD (or unit) concentration was $10^{-4} \mathrm{M}$ exceeding the TNS concentration of $10^{-5} \mathrm{M}$, so that most of TNS molecules added were bound to $\beta$-CD.

TNS alone in buffer showed negligible fluorescence. In the presence of $\beta$-CD, TNS showed some fluorescence, and in the presence of the polymer, as reported previously, ${ }^{5}$ a much greater fluorescence was observed with a significant blue shift. On addition of $(\beta-\mathrm{CD})_{2} \mathrm{G}$, which is considered to represent the local model of the polymer, a considerably larger fluorescence enhancement than $\beta$-CD was observed. In the case of $(\beta-C D)_{2} S$, a greater fluorescence than $(\beta-\mathrm{CD})_{2} \mathrm{G}$ was observed. It should be noted that mono- $\beta$-CD glutarate caused a smaller fluorescence enhancement than $\beta$-CD.

The interaction of the dimers with TNS was studied quantitatively by fluorescence titration at the fixed TNS concentration of $10^{-5} \mathrm{M}$. The effect of the

Table I. Characterization of CD-dimers

\begin{tabular}{lll}
\hline & \multicolumn{1}{c}{$(\beta-\mathrm{CD})_{2} \mathrm{G}$} & \multicolumn{1}{c}{$(\beta-\mathrm{CD})_{2} \mathrm{~S}$} \\
\hline $\mathrm{TLC}^{\mathrm{a}} R_{\mathrm{f}}$ & 0.08 & 0.08 \\
$\mathrm{IR}(\mathrm{KBr})$ & $1730 \mathrm{~cm}^{-1}$ & $1730 \mathrm{~cm}^{-1}$ \\
$\mathrm{NMR}\left(\mathrm{D}_{2} \mathrm{O}\right)$ & $\delta 2.35\left(\mathrm{p}, 2 \mathrm{H},-\mathrm{CH}_{2}-\right)$ & $\delta 3.28\left(\mathrm{~s}, 4 \mathrm{H},-\mathrm{CH}_{2}-\right)$ \\
& $\delta 3.00\left(\mathrm{t}, 4 \mathrm{H},-\mathrm{CH}_{2}-\right)$ \\
Anal. Found: & $\mathrm{C}, 44.82: \mathrm{H}, 6.29$ & $\mathrm{C}, 45.50 ; \mathrm{H}, 6.39 \%$ \\
Calcd: & $\mathrm{C}, 45.18 ; \mathrm{H}, 6.13$ & $\mathrm{C}, 44.94 ; \mathrm{H}, 6.09 \%$ \\
M.W. & $2,100 \pm 200$ & $2,200 \pm 100$ \\
Calcd: & 2,366 & 2,352 \\
\hline
\end{tabular}

\footnotetext{
${ }^{a}$ Solvent, $\mathrm{BuOH}: \mathrm{EtOH}: \mathrm{H}_{2} \mathrm{O}=2: 1: 1(\mathrm{v} / \mathrm{v} / \mathrm{v}), R_{\mathrm{f}}$ for $\beta-\mathrm{CD}=0.30$.

b VPO method.
}

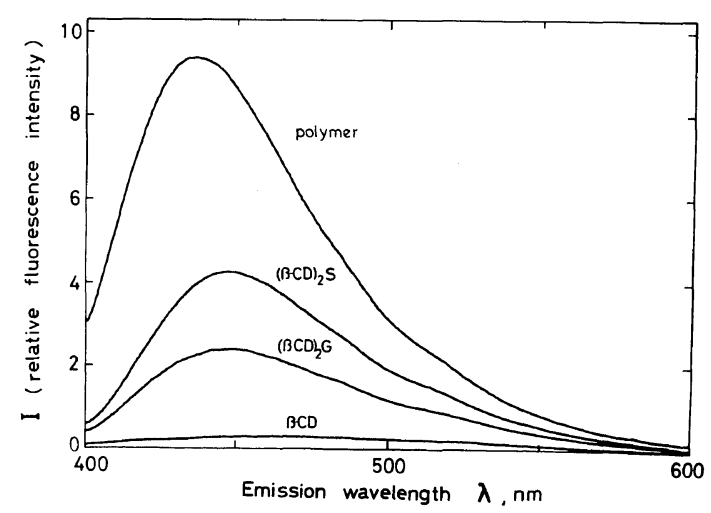

Figure 1. Fluorescence spectra of TNS in the presence of $\beta$-CD derivatives: $[\mathrm{TNS}]=1.00 \times 10^{-5} \mathrm{M},[\beta-\mathrm{CD}$ unit $]=1.00 \times 10^{-4} \mathrm{M}$.

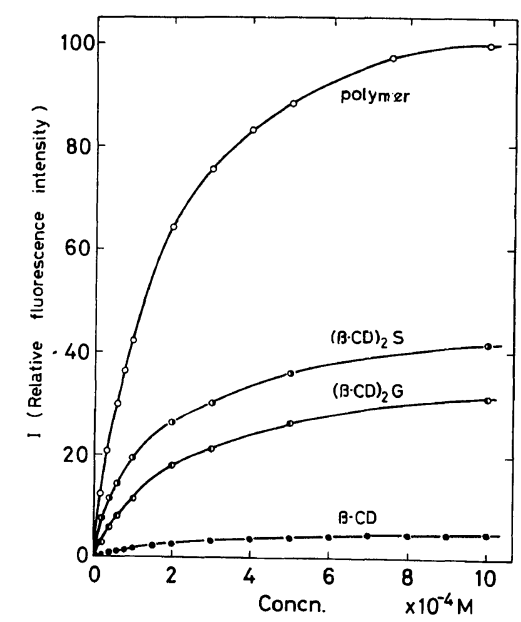

Figure 2. The effect of concentration of $\beta-C D$ derivatives on fluorescence intensity of TNS: $[\mathrm{TNS}]=1.00 \times 10^{-5} \mathrm{M}$.

concentration of $\beta$-CD unit on the fluorescence intensity is shown in Figure 2. The dimers caused a considerably larger increase in fluorescence than did $\beta$-CD throughout the concentration range tested, while the polymer caused even a larger increase in the fluorescence than the dimers. $(\beta-\mathrm{CD})_{2} \mathrm{~S}$ showed a larger effect than $(\beta-\mathrm{CD})_{2} \mathrm{G}$. The intensity showed saturation with an increase in the $\mathrm{CD}$ concentration, indicating that the fluorescence change was caused by the formation of a complex.

Figure 3 shows the double reciprocal plots of the results of fluorescence titration. If the binding sites 


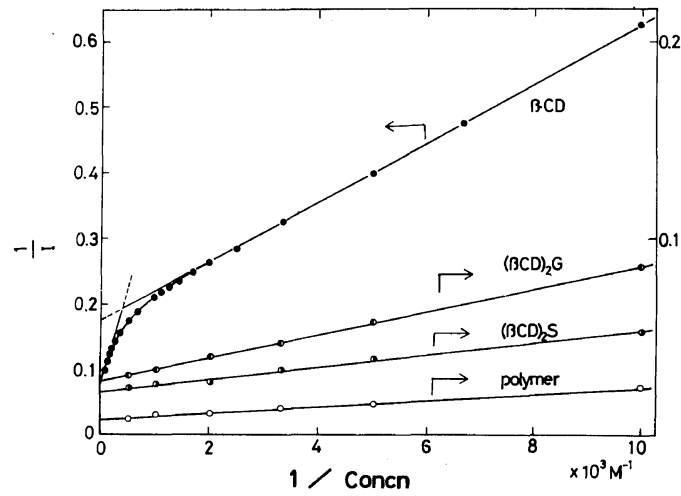

Figure 3. Double reciprocal plots for titration of TNS by $\beta$-CD derivatives: $[\mathrm{TNS}]=1.00 \times 10^{-5} \mathrm{M}$.

are homogeneous and independent, the double reciprocal plot should give a straight line. ${ }^{9}$ The plots for the dimers are linear, as are those for the polymer, indicating the homogeneity of the binding sites. However, the plot for $\beta$-CD comprise two straight lines, indicating the existence of two kinds of complexes. ${ }^{5}$

The emission maximum $\left(\lambda_{\max }^{\mathrm{F}}\right)$ of TNS changes when formation of complexes take place. The effect of the concentration of the $\beta$-CD unit on the wavelength of the maximal emission of TNS is shown in Figure 4. The $\lambda_{\max }^{\mathrm{F}}$ with $(\beta-\mathrm{CD})_{2} \mathrm{G}$ remained constant at $447 \mathrm{~nm}$ throughout the concentration range examined as well as that with the polymer at $438 \mathrm{~nm}$. The $\lambda_{\max }^{\mathrm{F}}$ of the $\beta$-CD-TNS

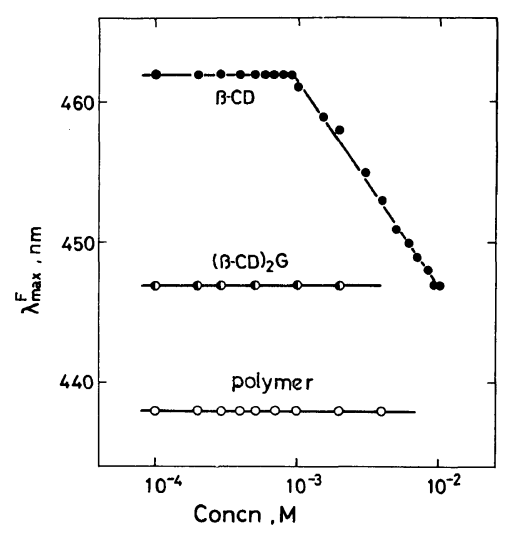

Figure 4. The effect of concentration of $\beta-C D$ derivatives on the wavelength of maximal emission of TNS: $[\mathrm{TNS}]=1.00 \times 10^{-5} \mathrm{M}$. system is constant at $462 \mathrm{~nm}$ until the $\beta$-CD concentration reaches $10^{-3} \mathrm{M}$; it then gradually shifts to a shorter wavelength. These results indicate that a stepwise complex formation occurs for $\beta-\mathrm{CD}$, whereas a homogeneous binding occurs for the dimers as well as for the polymer.

The maximum emission of both dimer complexes is $447 \mathrm{~nm}$ which is similar to that of the $2: 1$ complex of $\beta$-CD. This result may indicate that the polarity of the environment ${ }^{10-13}$ of the dimer complexes is similar to that of the $2: 1$ complex of $\beta$-CD. The fluorescence intensity of the dimer complexes is even stronger than that of $\beta-C D$, suggesting that the dimer complexes are perhaps more rigid ${ }^{14,15}$ than the $2: 1$ complex of $\beta$-CD.

The polymer complex showed a larger blue shift and a much larger fluorescence enhancement than the dimer complexes. The polymer complex may assume a more rigid conformation ${ }^{13,14}$ than the dimers and may exclude more effectively the solvent relaxation ${ }^{13,16}$ presumably because of deeper inclusion. In fact, examination of molecular models reveals that $\mathrm{CDs}$ in the dimers have more conformational freedom than those in the polymer where extremely bulky $\mathrm{CD}$ units considerably restrict the freedom each other.

\section{Stoichiometry}

The stoichiometry was determined by the continuous variation method. Figure 5 shows the continuous variation plot for the $(\beta-\mathrm{CD})_{2} \mathrm{G}-\mathrm{TNS}$ system. In this concentration range, the plot for the $\beta$ -

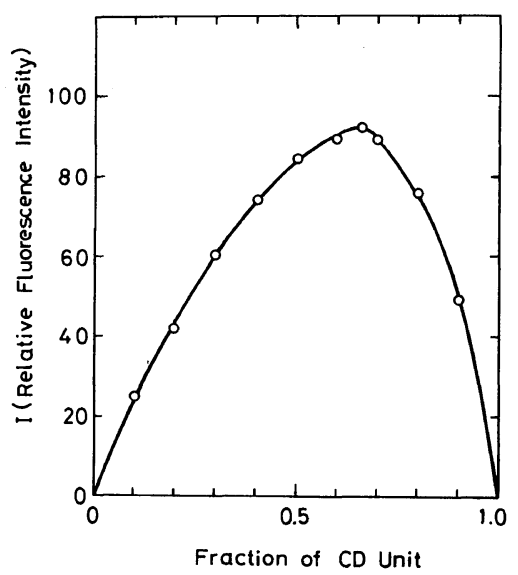

Figure 5. Continuous variation plot of $(\beta-\mathrm{CD})_{2} \mathrm{G}$ -TNS system: $[\beta-\mathrm{CD}$ unit $]=1.00 \times 10^{-4} \mathrm{M}$. 
CD system showed a maximum at a molar fraction of 0.5 , indicating $1: 1$ inclusion. ${ }^{5}$ On the other hand, the plot for the $(\beta-C D)_{2} \mathrm{G}$ system showed a maximum at 0.66 which corresponds to $2: 1$ (CD:TNS) stoichiometry. These results indicate that two $\beta$-CD units of the dimer participate in the binding of one TNS molecule. The same results were obtained in the cases of $(\beta-C D)_{2} \mathrm{~S}$ and the polymer.

The dissociation constants and fluorescence properties of the complexes of TNS with $\beta$-CD, the dimers, and the polymer are summarized in Table II. At low concentrations, as reported previously, ${ }^{5} \beta$ $\mathrm{CD}$ forms a $1: 1$ inclusion complex but it forms a $2: 1$ complex at higher concentrations. The fluorescence intensity of the $2: 1$ complex is stronger than that of the $1: 1$ complex and the maximum emission of the $2: 1$ complex is at a shorter wavelength than that of the $1: 1$ complex.

In the case of the dimers, only the $2: 1$ complexes are formed. The dissociation constant of the $(\beta$ CD) ${ }_{2} \mathrm{G}$ complex is $1.2 \times 10^{-4} \mathrm{M}$. The $2: 1$ complex is 400 -times more stable than that of $\beta$-CD. $(\beta-C D)_{2} \mathrm{~S}$ forms a $2: 1$ complex which is twice as stable as that of $(\beta-C D)_{2} \mathrm{G}$. This difference may be due to the slight variation in the rigidity of the complex. The maximum emission of the dimer complexes is $447 \mathrm{~nm}$ which is the same as that of the $2: 1$ complex of $\beta$ $\mathrm{CD}$. The fluoresecence intensity of the dimer complexes is even stronger than that of $\beta$-CD.

The polymer also forms only a $2: 1$ complex with TNS and the complex is much more fluorescent than the $2: 1$ complex of $\beta$-CD. The dissociation constant of the polymer-TNS complex is also similar to those of the dimers.

Molecular models of $\beta$-CD and TNS indicate that

Table II. Dissociation constants and fluorescence properties of complexes of TNS with $\beta$-CD derivatives

\begin{tabular}{lccrc}
\hline & CD:TNS & $K_{\mathrm{d}} / \mathrm{M}$ & $\begin{array}{r}\lambda_{\mathrm{m}}{ }^{\mathrm{F}} \\
\mathrm{nm}\end{array}$ & $\begin{array}{c}\text { Relative } \\
\text { fluorescence } \\
\text { intensity }\end{array}$ \\
\hline$\beta$-CD & $1: 1$ & $2.5 \times 10^{-4}$ & 462 & 1 \\
& $2: 1$ & $5 \times 10^{-2 \mathrm{a}}$ & $\leqslant 447$ & 3 \\
$(\beta \text {-CD })_{2} \mathrm{G}$ & $2: 1$ & $1.2 \times 10^{-4}$ & 447 & 8 \\
$(\beta \text {-CD })_{2} \mathrm{~S}$ & $2: 1$ & $0.6 \times 10^{-4}$ & 447 & 9 \\
Polymer & $2: 1$ & $1.0 \times 10^{-4}$ & 438 & 20 \\
\hline
\end{tabular}

a A pair of CD molecules are treated as a unit. ${ }^{9}$

b Calculated from $I_{\infty}$ of Figure 3. ${ }^{9}$
TNS is too large to be completely included in a single $\beta$-CD cavity which can accomodate only one aromatic ring: either the toluidinyl group or the naphthalene ring can enter the $\beta$-CD cavity. At first, one of these can be included in the $\beta$-CD cavity, and then the other can associate with the second CD molecule to form the $2: 1$ complex. Such a ternary complex may be formed only in the presence of a large excess of $\beta$-CD. In the case of the dimers, two CDs are located close to each other. When the first one of the aromatic groups is included, the other can follow easily, resulting in the formation of the $2: 1$ complex. In a space-filling model, the TNS molecule appears to fit well into the two $\beta$-CD cavities.

The high stability of the dimers-TNS complexes as compared to the $\beta$-CD complex indicates that both the toluidinyl group and the naphthyl group are included in two neighboring CDs cooperatively. Since two CDs are linked by the chain, the cooperation makes dissociation of the $2: 1$ complex difficult. The high stability of the polymer-TNS complex can be explained by the cooperation effect of two neighboring $\beta$-CDs as observed for the dimer complexes.

A large number of studies on the CD-substrate complexes have assumed a $1: 1$ stoichiometry. ${ }^{4}$ The $2: 1$ stoichiometry for $\mathrm{CD}$ : substrate is suggested for methyl orange, ${ }^{17}$ TNS, ${ }^{18}$ 4-biphenylcarboxylate, ${ }^{19}$ and heptane ${ }^{20}$ Molecular recognition by inclusion is an important process in biological systems. Recently molecules with complementary structures have been designed to identify substrate molecules. ${ }^{21,22}$ Molecules with two binding sites have been synthesized. Bis(crown ether), for example, forms much more stable complexes with metal cations than the corresponding monomers. ${ }^{23}$ In our study, two CD units linked by the chain were found to cooperate in binding a large substrate to the chain. It is important to arrange binding sites in such a way as to enhance the stability and to achieve better selectivity.

\section{EXPERIMENTALS}

\section{Materials}

$\beta$-CD was kindly supplied from Hayashibara Biochemical Inc. and was purified as described previously. ${ }^{3}$ Poly(acryloyl- $\beta$-CD) was prepared as described previously. ${ }^{3}$ TNS was purchased from Sigma Chemical Co. and its purity was checked by thin layer chromatography on silica gel $\mathrm{G}$. $\operatorname{Bis}(m$ - 
nitrophenyl) succinate and glutarate were prepared from $m$-nitrophenol and succinic acid using dicyclohexyl carbodiimide (DCC) by a procedure similar to that described for $p$-nitrophenyl carboxylates. ${ }^{4}$

$(\beta-\mathrm{CD})_{2} \mathrm{~S}$. $\beta$-CD $(11.5 \mathrm{~g}, 0.01 \mathrm{~mol})$ was dissolved in 1 liter of carbonate buffer, $\mathrm{pH} 9.1$, and this was mixed all at one time with $10 \mathrm{ml}$ of an acetonitrile solution of bis( $m$-nitrophenyl) succinate $(0.36 \mathrm{~g}$, $0.01 \mathrm{~mol})$. The reaction mixture was shaken vigorously for $5 \mathrm{~min}$, brought to $\mathrm{pH} 3$ with dilute $\mathrm{HCl}$ to terminate the reaction, and cooled in an ice bath. The unreacted acrylate crystallized out and was filtered off. The resultant filtrate was dialyzed against distilled water for 3 to 5 days to remove low molecular weight compounds ( $m$-nitrophenol and sodium chloride). Alternatively the filtrate was ultrafiltrated by a Amicon UM-10 UF membrane. Then the resulting solution was condensed by evaporation and the residue was subjected to gel chromatography on a Sephadex G-15 column $(2.5 \times 100 \mathrm{~cm})$ with water to separate the unreacted $\beta$-CD. Elution was followed by measuring the $\mathrm{CD}$ concentration by the phenol-sulfuric acid method, showing 3 peaks, the dimer, the monofunctional by-product and $\beta$ $\mathrm{CD}$. The yield was about $5 \%$ calculated from $\operatorname{bis}(m-$ nitrophenyl) succinate.

$(\beta-\mathrm{CD})_{2} \mathrm{G}$ was prepared by a similar method to that of $(\beta-\mathrm{CD})_{2} \mathrm{~S}$. The crude product was subjected to a Biogel P-2 column $(2.5 \times 80 \mathrm{~cm})$ with water eluent, and was separated into two peaks, the dimer and the monofunctional by-product. The yield was about $5 \%$ from bis( $m$-nitrophenyl) glutarate.

\section{Fluorescence Measurements}

Fluorescence measurements were performed in a $0.1 \mathrm{M}$ phosphate buffer ( $\mathrm{pH}$ 5.9) using a Union Giken FS-401 spectrofluorometer. The excitation wavelength was $366 \mathrm{~nm}$, and the fluorescence intensity was monitored at $460 \mathrm{~nm}$ for $\beta-\mathrm{CD}$, at $447 \mathrm{~nm}$ for the dimers, and $438 \mathrm{~nm}$ for the polymer; these were the corresponding emission maxima. The band width for the excitation was $8 \mathrm{~nm}$ and was usually $10 \mathrm{~nm}$ for emission. The concentration of TNS was determined spectrophotometrically, assuming that the molecular absorptivity $\varepsilon_{\mathrm{TNS}}=4300$ at $366 \mathrm{~nm}$. Absorption spectra were measured using a Hitachi spectrometer Model 124.

\section{Determination of Dissociation Constants}

The dissociation constants were determined as described previously except that the concentration of the dimer was used in place of the concentration of the CD unit. $^{5}$

Acknowledgement. This work was supported by a Grant-in-Aid for Special Project Research from the Ministry of Education. We are indebted to Hayashibara Biochemical Inc. for their generous supply of $\beta$-cyclodextrin.

\section{REFERENCES}

1. J. H. Fendler and E. J. Fendler, "Catalysis in Micellar and Macromolecular Systems," Academic Press, New York, N.Y., 1975.

2. M. L. Bender and M. Komiyama "Cyclodextrin Chemistry," Springer Verlag, Basel, 1978.

3. A. Harada, M. Furue, and S. Nozakura, Macromolecules, 9, 701 (1976).

4. A. Harada, M. Furue, and S. Nozakura, Macromolecules, 9, 705 (1976).

5. A. Harada, M. Furue, and S. Nozakura, Macromolecules, 10, 676 (1977).

6. L. Stryer, Science, 162, 526 (1968).

7. L. Brand and J. R. Gohlke, Ann. Rev. Biochem., 41, 843 (1972).

8. R. L. Van Etten, G. A. Clowes, J. F. Sebastian, and M. L. Bender, J. Am. Chem. Soc., 89, 3253 (1967).

9. I. M. Klotz and D. L. H. Hunston, Biochemistry, 10, 3065 (1971).

10. C. J. Selisker and L. Brand, Science, 171, 799 (1971).

11. E. M. Kosower and H. Dodiuk, Chem. Phys. Lett., 26, 545 (1974).

12. E. M. Kosower, H. Dodiuk, K. Tanizawa, M. Ottolenghi, and N. Orbach, J. Am. Chem. Soc., 97, 2167 (1975).

13. G. R. Fleming, G. Porter, R. J. Robbins, and J. A. Synowic, Chem. Phys. Lett., 52, 228 (1977).

14. W. O. McClure and G. H. Edelman, Biochemistry, 5, 1908 (1966).

15. A. Ainworth and M. T. Flanagan, Biochim. Biophys. Acta, 194, 213 (1969).

16. C. J. Selisker and L. Brand, J. Am. Chem. Soc., 93, 5405, 5414 (1971).

17. K. Harata, Bull. Chem. Soc. Jpn., 49, 1493 (1976).

18. H. Kondo, H. Nakatani, and K. Hiromi, J. Biochem., 79, 393 (1976).

19. R. I. Gelb, I. M. Schwartz, C. T. Murray, and D. A. Laufer, J. Am. Chem. Soc., 100, 3553 (1978).

20. A. Wishnia and S. J. Lappi, J. Mol. Biol., 82, 77 (1974).

21. D. J. Cram and J. M. Cram, Acc. Chem. Res., 11, 8 (1978).

22. J. M. Lehn, Acc. Chem. Res., 11, 49 (1978).

23. M. Bourgoin, K. H. Wong, J. Y. Hui, and J. Smid, J. Am. Chem. Soc., 97, 3462 (1975). 